\title{
A review of aluminium phosphide poisoning and a flowchart to treat it: recommendation for a revision
}

\author{
Seyed Mostafa Mirakbari \\ Qazvin University of Medical Sciences, Qazvin, Iran
}

I read with great interest the paper entitled "A review of aluminium phosphide poisoning and a flowchart to treat it" published in your journal by Hashemi-Domeneh et al. (1). This article has tried, and failed, to review up-to-date knowledge related to aluminium phosphide (ALP) poisoning and propose an easy-to-use management paradigm for health professionals. In addition, it has largely ignored the existing evidence of the different aspects of ALP poisoning beyond metabolism-based consequences. It has therefore failed to answer the question why the mortality rate remains high, even with such considerable advances in treatment protocols.

First, the paper has undermined the significance of local thermal injuries in different settings of ALP poisoning. These complications have frequently been reported in the literature and are believed to play a substantial role in the unfavourable outcome (2). Second, the paper is not consistent with two of the three pillars of evidence-based medicine, including clinical expertise and best available evidence (3).

Expert opinions and current evidence propose three readily available and feasible recommendations that should be included in the flowchart described in this paper. These are as follows:

(A) Do not administer potassium permanganate in gastrointestinal (GI) decontamination. It induces an exothermic reaction that leads to burning and local thermal injuries, resulting in haemorrhages, hypotension, and death $(2,4-5)$. In addition, it is a strong oxidising agent that causes hemolysis and methemoglobinemia, which contribute to the deterioration of the course of the patient's recovery (6).

(B) Do not suction and lavage via a nasogastric tube, as it increases negative pressure in the stomach, which, in turn, provokes internal gas ignition $(2,7-8)$.

(C) Administer an intravenous (IV) lipid emulsion (5). The circulating toxic phosphine is trapped in a blood lipid pool, thereby hindering its site toxic effects.

I thank you for your attention to my viewpoints.
Conflict of interests

Author declares no conflict of interests.

\section{REFERENCES}

1. Hashemi-Domeneh B, Zamani N, Hassanian-Moghaddam H, Rahimi M, Shadnia S, Erfantalab P, Ostadi A. A review of aluminium phosphide poisoning and a flowchart to treat it. Arh Hig Rada Toksikol 2016;67:183-93. doi: 10.1515/ aiht-2016-67-2784

2. Mirakbari SM. Hot charcoal vomitus in aluminum phosphide poisoning - A case report of internal thermal reaction in aluminum phosphide poisoning and review of literature. Indian J Anaesth 2015;59:433-6. doi: 10.4103/00195049.160952

3. Masic I, Miokovic M, Muhamedagic B. Evidence based medicine - new approaches and challenges. Acta Inform Med 2008;16:219-25. doi: 10.5455/aim.2008.16.219-225

4. Senthilkumaran S, Ananth C, Menezes RG, Thirumalaikolundusubramanian P. Aluminium phosphide poisoning: Need for revised treatment guidelines. Indian J Anaesth 2015;59:831-2. doi: 10.4103/0019-5049.171624

5. Baruah U, Sahni A, Sachdeva HC. Successful management of aluminium phosphide poisoning using intravenous lipid emulsion: Report of two cases. Indian J Crit Care Med 2015;19:735-8. doi: 10.4103/0972-5229.171412

6. Sanaei-Zadeh H. Aluminum phosphide poisoning and development of hemolysis and methemoglobinemia. Indian J Crit Care Med 2012;16:248-9. doi: 10.4103/09725229.106519

7. Akinci E, Kocasaban DU, Vural K, Coskun F. Secondary intoxication of emergency department personnel with a flammable and highly toxic gas: A lethal aluminum phosphide poisoning case. Hong Kong J Emerg Med 2012;19:54-7.

8. Wahab A, Rabbani MU, Wahab S, Khan RA. Spontaneous self-ignition in a case of acute aluminium phosphide poisoning. Am J Emerg Med 2009;27:752.e5-6. doi: 10.1016/j.ajem.2008.09.045 